\title{
CUIDADOS DE ENFERMAGEM PARA A INCLUSÃO SOCIAL DA PESSOA COM DEFICIÊNCIA FÍSICA ADQUIRIDA: REVISÃO INTEGRATIVA.
}

\author{
NURSING CARE FOR SOCIAL INCLUSION OF PEOPLE WITH ACQUIRED PHYSICAL DISABILITIES: AN INTEGRATIVE REVIEW. \\ ATENCIÓN DE ENFERMERÍA PARA LA INCLUSIÓN SOCIAL DE PERSONAS CON DISCAPACIDAD FÍSICA ADQUIRIDA: REVISIÓN \\ INTEGRADORA.
}

DOI 10.33194/rper.2020.v3.n2.13.5827 | Submetido 11/11/2020 | Aprovado 15/12/2020

\author{
Rute Salomé da Silva Pereira ${ }^{1,2}$; Maria Manuela Martins ${ }^{2} \mathbb{D}$; Wiliam César Alves Machado ${ }^{3}$; Ana Isabel \\ Pereira ${ }^{4}\left(\mathbb{D}\right.$; Ana Maria da Silva Pereira ${ }^{5}$; ; Fabíola Hermes Chesani ${ }^{6}(\mathbb{D}$ \\ 1 - Instituto de Ciências Biomédicas Abel Salazar; 2 - Escola Superior de Enfermagem do Porto; 3 - Universidade Federal do \\ Estado do Rio de Janeiro - UNIRIO; 4 - Hospital Lusíadas, Porto; 5 - IPO, Porto; 6 - Universidade do Vale do Itajaí
}

\section{RESUMO}

Introdução: Os cuidados de enfermagem adequados podem promover a inclusão social da pessoa com deficiência física adquirida porque permitem a participação plena nas várias esferas da vida em sociedade, como no trabalho, saúde e lazer.

Objetivo: Analisar quais os cuidados de enfermagem desenvolvidos para a inclusão e participação social da pessoa com deficiência física adquirida.

Metodologia: Revisão integrativa da literatura. A pesquisa realizou-se nas bases de dados Web of Science, Scopus, no agregador de bases EBSCOhost e Biblioteca Virtual em Saúde, identificando-se estudos de 2010-2020.

Resultados: dos 615 estudos identificados incluíram-se 14 estudos demonstrando que alguns cuidados de enfermagem desenvolvidos são: a identificação de barreiras arquitetónicas e a promoção de transições de cuidado seguras e eficazes através do empoderamento da pessoa com deficiência.

Conclusão: A inclusão social das pessoas com deficiência física adquirida deve ser uma área de intervenção específica dos enfermeiros.

Palavras-chave: participação social; cuidados de enfermagem; pessoas com deficiência; revisão

\section{RESUMEN}

Introdución: La atención de enfermería adecuada puede promover la inclusión social de las personas con discapacidad adquirida porque permiten la participación plena en los distintos ámbitos de la vida en sociedad, como el trabajo, la salud y el ocio.

Objetivo: Analizar la atención de enfermería desarrollados para la inclusión y participación social de la persona con discapacidad física adquirida.

Metodología: Revisión integrativa de la literatura. Las búsquedas se realizó en las bases de datos Web of Science, Scopus, en el agregador EBSCOhost y Biblioteca Virtual em Saúde, identificando estudios de 2010-2020.

Resultados: De los 615 estudios identificados, se incluyeron 14 estudios que demuestran que algunos de los cuidados de enfermería desarrollados son: la identificación de barreras arquitectónicas y la promoción de transiciones asistenciales seguras y efectivas a través del empoderamiento de las personas con discapacidad.

Conclusión: La inclusión social de las personas con discapacidad adquirida debe ser un área de intervención específica para las enfermeras.

Palabras clave: participación social; atención de enfermería; personas con discapacidad; revisión

\section{ABSTRACT}

Introduction: Adequate nursing care can promote the social inclusion of people with acquired physical disabilities because it allows full participation in different areas of life in society, such as work, health and leisure."

Objective: To analyze which nursing care were developed for the inclusion and social participation of people with acquired physical disabilities.

Methodology: Integrative literature review. The research was conducted in the databases of Web of Science,Scopus, in databse aggregator EBSCOhost and Biblioteca Virtual em Saúde, identifying studies from 2010-2020. 
Results: of the 615 studies identified, 14 studies were included demonstrating that some developed nursing care are: the identification of architectural barriers and the promotion of safe and effective care transitions through the empowerment of people with disabilities.

Conclusion: The social inclusion of people with acquired physical disabilities must be a specific intervention in nursing care.

Keywords: social participation; nursing care; disabled person; review

\section{INTRODUÇÃO}

Em 2019 completou-se uma década desde que a República Portuguesa, através da Resolução da Assembleia da República $n^{\circ}$ 56/2009 de 30 de Julho, ratificou a Convenção das Nações Unidas sobre os Direitos das Pessoas com Deficiência, tornando-se assim um dos Estados Partes signatário da referida Convenção. (1)

Os objetivos da Convenção são promover, proteger e garantir todos os direitos humanos e liberdades fundamentais para todas as pessoas com deficiência e, o artigo $3^{\circ}$ refere como princípios gerais o respeito pela dignidade, autonomia e liberdade individual, o respeito pela diferença e aceitação das pessoas com deficiência como parte da diversidade humana, assim como, a participação e inclusão plena e efetiva na sociedade. (1)

A inclusão social pode ser definida, não apenas como o acesso em iguais condições como as demais pessoas, mas que se trata de uma participação total e plena nas diversas esferas da vida em sociedade. (2)

Para a inclusão social da pessoa com deficiência é necessário o sentimento de pertença a um grupo, que a pessoa se sinta aceite como um ser individual e possua relações interpessoais recíprocas, participando em diversas atividades como educação, cultura e lazer, cumprindo com os deveres fundamentais e obrigações devidas a todos os cidadãos e não apenas o usufruto dos direitos, num processo de participação ativa na sociedade. $(3,4)$

Os profissionais de saúde, nomeadamente os enfermeiros têm um papel social que deve ser desempenhado na prestação dos cuidados à pessoa com deficiência física adquirida, uma vez que acrescentam benefícios ao processo de reabilitação na recuperação, não apenas das capacidades funcionais, mais ainda da autonomia individual. $(5,6)$

As medidas políticas de anti exclusão e estratégias práticas que promovam a participação das pessoas com deficiência na sociedade são necessárias (3) uma vez que muitas pessoas relatam dificuldades na participação em eventos locais, festivais ou reuniões da comunidade, essencialmente em virtude de dificuldades na locomoção física conduzindo a que algumas se afastem de algum grupo social do qual anteriormente participava. (2)

No Recenseamento da População em 2001 apurou-se que um total de 636059 pessoas apresentavam deficiência, sendo que 156246 possuíam uma deficiência motora (7) e, em 2011 a principal dificuldade declarada foi a limitação no andar com uma expressão de $25 \%$ das respostas nas pessoas com cinco ou mais anos e, nas pessoas com 65 ou mais anos de $27 \%$. (8)

Com o fenómeno do envelhecimento, assistimos a uma maior expressão da deficiência na pessoa idosa, uma vez que, devido ao declínio funcional, estas adquirem uma deficiência física. $(2,9)$

A deficiência é assim um fenómeno complexo, dinâmico e multidimensional, que engloba diversos conceitos, tais como, funcionalidade e incapacidade, limitação na atividade e restrição na participação, em interação com fatores contextuais. $(10,11)$

Assim, uma pessoa com deficiência física adquirida pode vivenciar uma restrição na participação, que são problemas que a pessoa enfrenta no envolvimento em situações reais da vida. Este envolvimento associado à participação implica que a pessoa tome parte, seja incluída e participe em determinada área da vida e se sinta aceite ou, tenha acesso a recursos de que necessita. (10)

A complexidade da vida, saúde, doença e dos processos de reabilitação das pessoas com deficiência adquirida, têm características gerais e singulares. Porém, os enfermeiros estão presentes ao longo das várias fases do processo, desde os serviços de emergência e urgência, passando pelos centros e serviços especializados em reabilitação culminando nos cuidados de longo prazo, ou seja, na comunidade. (12)

Portillo e Cowley (13) referem que os enfermeiros são os profissionais que assumem uma posição estratégica para o desenvolvimento dos programas de reabilitação individualizada uma vez que são estes profissionais que passam mais tempo com os doentes e seus familiares, conseguem estabelecer relações mais próximas e de confiança, na sua prática diária lidam com respostas emocionais e vulnerabilidade, num contexto de profunda intimidade e têm as competências necessárias para avaliar, planear e implementar cuidados holísticos de acordo com as necessidades de cada pessoa e família.

Como destacado por Alvarez, Machado (14), compete à equipa de reabilitação, especificamente aos enfermeiros de reabilitação, investir na inclusão dessas pessoas na sociedade, mostrando as possibilidades de viver com plenitude, no trabalho, saúde, lazer, não obstante as limitações funcionais decorrentes da lesão neurológica e deficiência adquirida.

Face ao contexto teórico exposto foi elaborada a seguinte questão orientadora desta revisão integrativa da literatura: "Que cuidados de enfermagem são desenvolvidos para a inclusão social da pessoa com deficiência física adquirida?", com o objetivo de analisar os cuidados de enfermagem desenvolvidos para 
a inclusão e participação social da pessoa com deficiência física adquirida.

\section{MÉTODOS}

Efetuámos um estudo de revisão integrativa da literatura, no sentido de delimitar o problema de investigação, compreendendo amplamente o fenómeno em estudo e, sintetizar o conhecimento existente sobre a temática em estudo. (15)

Esta revisão integrativa desenvolveu-se através das seguintes etapas: identificação do tema e seleção da questão de pesquisa; estabelecimento de critérios para inclusão e exclusão de estudos; definição das informações a serem extraídas dos estudos selecionados; avaliação dos estudos incluídos na revisão integrativa; interpretação dos resultados e, apresentação da revisão integrativa. (15)

Como estratégia de interrogação para a formulação da questão orientadora utilizámos a estratégia PICO (População: pessoa com deficiência física adquirida; Intervenção: cuidados de enfermagem; Comparação: sem comparação e, Outcomes: promoção da inclusão e participação social). Para efeitos desta revisão cuidados de enfermagem são as intervenções autónomas ou interdependentes realizadas por enfermeiros segundo as suas qualificações profissionais, podendo estas intervenções ser diagnósticas, terapêuticas, preventivas, prognósticas, de avaliação e reformulação dessas mesmas intervenções numa interação entre enfermeiro e a pessoa, família, grupos e comunidade. (16)

Para a estratégia de pesquisa usámos uma estrutura coerente combinando os termos de busca, os operadores booleanos e os componentes da estratégia PICO.

A frase booleana foi escrita da seguinte forma: (("social inclusion" OR "social integration" OR "social participation" OR "community integration" OR "social rehabilitation") AND (disabilit* OR disabled OR disabling OR handicap* OR "mobility Limitation" OR locomotion OR "reduced mobility" OR impair*) AND (nurs* OR "nursing care" OR “nurse's role")).

Foram definidos critérios de inclusão/exclusão (Tabela 1) para a seleção dos estudos pretendidos face à questão formulada.

A estratégia de pesquisa foi desenvolvida em três passos. Inicialmente efetuámos uma pesquisa naturalista na base de dados Scopus e WOS para identificar palavras e termos controlados, bem como, linguagem específica da rede autoral. Posteriormente, realizámos uma segunda pesquisa utilizando as palavras-chave que foram definidas de acordo com os descritores indexados, nomeadamente os termos MeSH, descritores em Ciências da Saúde, e linguagem específica da rede autoral.
Tabela 5 - Critérios de inclusão e exclusão para a seleção dos estudos

\begin{tabular}{|l|l|l|}
\hline $\begin{array}{c}\text { Critérios } \\
\text { de seleção }\end{array}$ & \multicolumn{1}{|c|}{$\begin{array}{c}\text { Critérios de } \\
\text { inclusão }\end{array}$} & \multicolumn{1}{|c|}{$\begin{array}{c}\text { Critérios de } \\
\text { exclusão }\end{array}$} \\
\hline $\begin{array}{l}\text { Partici- } \\
\text { pantes }\end{array}$ & $\begin{array}{l}\text { Pessoa com } \\
\text { deficiência física } \\
\text { adquirida } \\
\text { Mais de } 18 \text { anos }\end{array}$ & $\begin{array}{l}\text { Outro tipo de } \\
\text { deficiência } \\
\text { Idade inferior a 18 anos }\end{array}$ \\
\hline Intervenção & $\begin{array}{l}\text { Cuidados de } \\
\text { enfermagem }\end{array}$ & $\begin{array}{l}\text { Cuidados prestados por } \\
\text { outros profissionais }\end{array}$ \\
\hline Outcomes & $\begin{array}{l}\text { Promoção da } \\
\text { inclusão e } \\
\text { participação social }\end{array}$ & $\begin{array}{l}\text { Resultados relativos a } \\
\text { exclusão e isolamento } \\
\text { social }\end{array}$ \\
\hline Desenho & Artigos originais & Revisões da literatura \\
estudo & & \\
\hline
\end{tabular}

Para a identificação dos estudos realizou-se, no dia 29 de Julho de 2020, uma busca nas bases de dados Web of Science (nomeadamente, Core Collection, Scielo Citation Index, Russian Citation Index, Corean Citation Index, Medline) e Scopus (Medline e EMBASE), no agregador de bases EBSCOhost (Academic Search Complete; Business Source Complete; CINAHL Complete; CINAHL Plus with Full Text; ERIC; Library, Information Science \& Technology Abstracts; MedicLatina; MEDLINE with Full Text; Psychology and Behavioral Sciences Collection;Regional Business News; SPORTDiscus with Full Text; MEDLINE) e na Biblioteca Virtual em Saúde (Base de dados: LILACS, IBECS, Index Psicologia - Periódicos técnico-científicos, BDENF Enfermagem, CUMED).

Por fim, alargámos a pesquisa a revistas específicas da área, nomeadamente a Revista Portuguesa de Enfermagem de Reabilitação e, também, analisámos as referências bibliográficas dos estudos selecionados com intuito de identificarmos potenciais estudos com relevância para a temática em estudo.

Foram aplicados limitadores de pesquisa, relativo ao idioma, sendo considerados para inclusão nesta revisão integrativa estudos redigidos em português, inglês e espanhol. No agregador de bases EBSCOhost, aplicámos o limitador relativo à idade, uma vez que este permitia essa opção.

Selecionados os estudos publicados no período entre 1 de Janeiro de 2010 e 31 Julho de 2020. O processo de seleção da literatura, extração dos dados e síntese do conhecimento foi efetuado por dois revisores independentes. Esta revisão foi conduzida segundo padrões éticos de rigor e integridade, desde o planeamento até à redação do relatório final e a divulgação de resultados.

Os dados foram extraídos dos artigos selecionados e organizados usando uma Matriz de Revisão em formato Excell permitindo efetuar uma síntese dos estudos e analisar os principais resultados extraindo detalhes específicos sobre as características dos participantes e da intervenção, e os resultados pertinentes dos outcomes avaliados. A síntese integrativa do conhecimento sobre os contributos para o cuidado em enfermagem prestados à pessoa com deficiência física 
adquirida para a promoção da sua inclusão foi agrupada por cuidados de enfermagem para a inclusão social.

\section{RESULTADOS}

A pesquisa identificou um total de 615 estudos. Destes, foram excluídos 348 por serem duplicados; dos restantes 267 pela análise do título e resumo foram excluídos 222 estudos. Dos restantes 45 artigos avaliados para elegibilidade foram excluídos 31 dado não cumprirem os critérios de inclusão após a leitura integral do texto. Integram o corpus de análise desta revisão 14 artigos, como apresentado no fluxograma PRISMA (Figura 1).

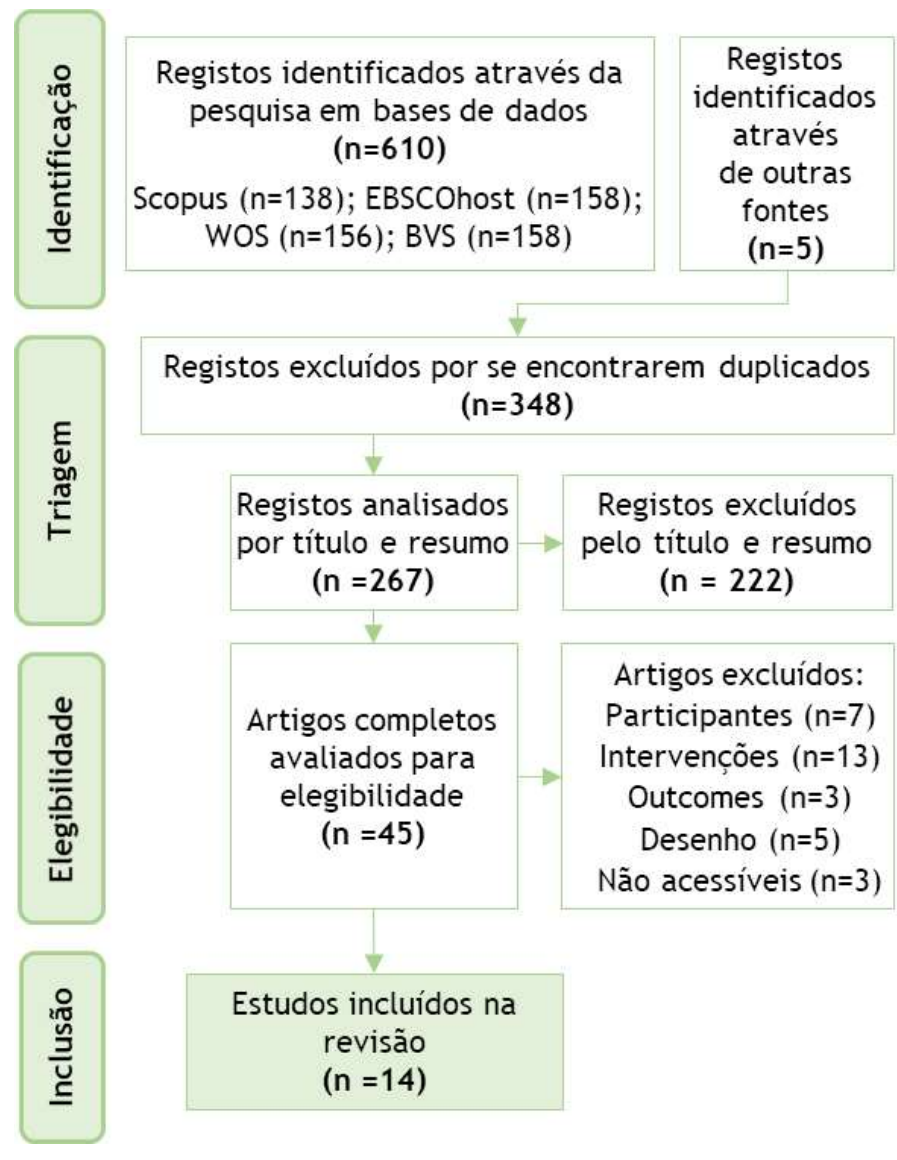

Figura 3. Fluxograma PRISMA - Processo de pesquisa e seleção de estudos

Fonte: Moher D, Liberati A, Tetzlaff J, Altman DG, The PRISMA Group (2009). Preferred Reporting Items for Systematic Reviews and MetaAnalyses: The PRISMA Statement. PLoS Med 6(7): e1000097. doi:10.1371/journal.pmed1000097

A amostra de estudos selecionados é heterogénea, com variabilidade no tipo de desenho de estudo. Quanto ao país em que o estudo foi desenvolvido, o Brasil ( $\mathrm{N}=4)$ e Portugal $(\mathrm{N}=4)$ concentram a maioria dos estudos, o que demonstra um interesse relativamente aos cuidados de enfermagem à pessoa com deficiência física adquirida tendo em vista a sua inclusão social. Os principais anos de publicação sobre a temática foram $2019(\mathrm{~N}=4), 2018$ $(\mathrm{N}=3)$ e $2011(\mathrm{~N}=3)$, como podemos constatar pela análise da Tabela 2.

Da avaliação qualitativa dos estudos apresentamos na Tabela 3 o resumo dos principais dados com interesse para a temática em estudo, por forma a facilitar a leitura e análise dos dados.
Para apresentar os cuidados de enfermagem para a inclusão social da pessoa com deficiência física adquirida que podem trazer sugestões e caminhos novos à pratica de enfermagem estas estão organizadas em cuidados de enfermagem para a inclusão social: identificar as pessoas com deficiência física adquirida; avaliar a capacidade funcional da pessoa com deficiência para a realização de atividades de vida e participação social; avaliar o impacto da alteração da capacidade funcional na qualidade de vida da pessoa; implementar cuidados de enfermagem baseado em modelos teóricos e no processo de enfermagem; identificar barreiras arquitetónicas nos vários contextos de vida da pessoa e orientar para a sua eliminação; implementar programas de treino motor e de reabilitação social; conceber planos de cuidados personalizados - Enfermeiro gestor de caso; promover e facilitar transições de cuidado seguras e eficazes através do empoderamento da pessoa com deficiência; contribuir para o desenvolvimento de políticas, melhorando assistência à pessoa com deficiência e melhorando os serviços comunitários / colaboração com o poder autárquico.

\section{DISCUSSÃO}

Nos últimos anos e, apesar da legislação em vigor e das competências dos enfermeiros, nomeadamente dos enfermeiros de reabilitação na promoção da cidadania, verifica-se uma insuficiente resposta dos cuidados de enfermagem para a inclusão social da pessoa com deficiência física adquirida. (17)

Foi neste sentido que desenvolvemos esta revisão integrativa da literatura com o objetivo de analisar quais são os cuidados de enfermagem desenvolvidos para a inclusão e participação social da pessoa com deficiência física adquirida no sentido de apontarmos orientações sustentadas para a prática de enfermagem. Os aspetos mais importantes que revestem cada um dos estudos incluídos, são apresentados seguidamente através de uma síntese descritiva dos mesmos.

Da análise dos vários estudos constatamos uma considerável heterogeneidade entre eles no que respeita às características da população em estudo e à natureza da intervenção dos cuidados de enfermagem desenvolvidos.

\section{Identificar as pessoas com deficiência física adquirida}

O conceito de deficiência é heterogéneo pelo que se torna essencial conhecer quem são as pessoas com deficiência física adquirida para a prestação de cuidados em enfermagem.

Tal como podemos notar nas características dos intervenientes em cada estudo podem ser pessoas com doença do foro neurológico, por exemplo Parkinson, AVC, esclerose múltipla $(13,18)$ ou com lesão vertebro medular. (19) Também idosos, dado que o processo de envelhecimento está intimamente relacionado com a perda de funcionalidade, fragilidade e dependência na realização de atividades de vida diárias, doenças crónicas e deficiência. (20-23) 
Tabela 6 - Identificação dos estudos para análise final

\begin{tabular}{|c|c|c|c|}
\hline Ano & País & Autores e Título & Desenho estudo \\
\hline 2019 & EUA & $\begin{array}{l}\text { Crews DC, Delaney AM, Walker Taylor JL, Cudjoe TKM, Nkimbeng M, Roberts L, et al. Pilot } \\
\text { Intervention Addressing Social Support and Functioning of Low Socioeconomic Status Older } \\
\text { Adults With ESRD: The Seniors Optimizing Community Integration to Advance Better Living } \\
\text { with ESRD (SOCIABLE) Study. }\end{array}$ & $\begin{array}{l}\text { Qualitativo; } \\
\text { Controlado } \\
\text { randomizado }\end{array}$ \\
\hline 2013 & Brasil & $\begin{array}{l}\text { França ISXd, Coura AS, Sousa FSd, Almeida PCd, Pagliuca LMF. Qualidade de vida em } \\
\text { pacientes com lesão medular. }\end{array}$ & Epidemiológico \\
\hline 2017 & Suécia & $\begin{array}{l}\text { Granbom } M \text {, Kristensson J, Sandberg } M \text {. Effects on leisure activities and social participation } \\
\text { of a case management intervention for frail older people living at home: a randomised } \\
\text { controlled trial. }\end{array}$ & $\begin{array}{l}\text { tal } \\
\text { ado. }\end{array}$ \\
\hline 2018 & Brasil & $\begin{array}{l}\text { Machado WCA, Pereira JdS, Schoeller SD, Júlio LC, Martins MMFPS, Figueiredo NMAd. } \\
\text { Integralidade na rede de cuidados da pessoa com deficiência. }\end{array}$ & Reflexivo \\
\hline 2019 & Brasil & $\begin{array}{l}\text { Nascimento VFd, Gonçalves RA, Jesus WGd, Graça BCd, Terças-Trettel ACP, Hattori TY. } \\
\text { Acessibilidade de Pessoas com Deficiência da Residência à Unidade de Saúde da Família. }\end{array}$ & \\
\hline 2020 & Irão & $\begin{array}{l}\text { Neiseh, F., Dalvandi, A., Tabrizi, K. N., Shahboulaghi, F. M., Fallahi-Khoshknab, M., \& } \\
\text { Shemshadi, H. Barriers and Facilitators to Emancipation Process in Persons with Physical } \\
\text { Disability-A Grounded Theory. }\end{array}$ & \\
\hline 2018 & Port & $\begin{array}{l}\text { Pereira, R. S. D. S., Martins, M. M.F.P.S., Gomes, B., Aguilera, J. A. L. A., \& Santos, J. A } \\
\text { intervenção do enfermeiro de reabilitação na promoção da acessibilidade. }\end{array}$ & $\begin{array}{l}\text { Quantit } \\
\text { descriti }\end{array}$ \\
\hline 2018 & Port & $\begin{array}{l}\text { Pereira, R. S. d. S., Martins, M. M. F. P. S., Gomes, B. P., Schoeller, S. D., Laredo-Aguilera, } \\
\text { J. A., Ribeiro, I., \& Cunha, P. As autarquias e a promoção da acessibilidade arquitetónica. }\end{array}$ & Q \\
\hline 2014 & Brasil & $\begin{array}{l}\text { Pires FRdO, Deitos BG, Schoeller SD, Santos SMAd, Vargas MAdO, Silva KM. Barreras y } \\
\text { facilitadores arquitectónicos: un desafío para la independencia funcional. }\end{array}$ & ivo, \\
\hline 2011 & Espanha & $\begin{array}{l}\text { Portillo, M. C., \& Cowley, S. Working the way up in neurological rehabilitation: The holistic } \\
\text { approach of nursing care. }\end{array}$ & Investigação-ação \\
\hline 2011 & Espanha & $\begin{array}{l}\text { Portillo, M. C., \& Cowley, S. Social rehabilitation in long-term conditions: Learning about } \\
\text { the process. }\end{array}$ & Inve \\
\hline 2019 & Portugal & $\begin{array}{l}\text { Silva, C. F. d., Oliveira, F. D. B. d., Prazeres, V. M. P., Ribeiro, M. P., \& Ribeiro, O. M. P. L. } \\
\text { Novos desafios para velhos problemas- O Enfermeiro Especialista em Enfermagem de } \\
\text { Reabilitação na Promoção da Acessibilidade. }\end{array}$ & R \\
\hline 2019 & Portugal & $\begin{array}{l}\text { Silva, C. S., Pacheco, M. N., Martins, M. M. F. P. S., Carvalho, A. E. C., Pereira, R. S. S., \& } \\
\text { Machado, W. C. A. M. Acessibilidade dos Edifícios destinados à prática de desporto nas } \\
\text { pessoas com deficiência. }\end{array}$ & $\begin{array}{l}\text { Quantitativ } \\
\text { descritivo, }\end{array}$ \\
\hline 2011 & Canadá & $\begin{array}{l}\text { St-Germain, D., Boivin, B., \& Fougeyrollas, P. The Caring-Disability Creation Process model: } \\
\text { a new way of combining 'Care' in nursing and 'Rehabilitation' for better quality of services } \\
\text { and patient safety. }\end{array}$ & \\
\hline
\end{tabular}

Tabela 7 - Resumo dos dados extraídos dos estudos incluídos na revisão

\begin{tabular}{|c|c|c|c|c|}
\hline Autores & Objetivos & Participantes & Resultados & Conclusões \\
\hline Crews et al. & $\begin{array}{l}\text { Implementar um estudo } \\
\text { piloto baseado num } \\
\text { programa para } \\
\text { promover } \\
\text { funcionalidade e } \\
\text { participação social de } \\
\text { idosos em tratamento } \\
\text { de hemodiálise no } \\
\text { domicílio. }\end{array}$ & $\begin{array}{l}1^{\text {a }} \text { fase: } 7 \text { idosos em } \\
\text { programa de } \\
\text { hemodiálise } \\
2^{\text {a }} \text { fase: Grupo de } \\
\text { intervenção e grupo } \\
\text { de controlo }(\mathrm{N}=12) \\
\text { (Single-blind) }\end{array}$ & 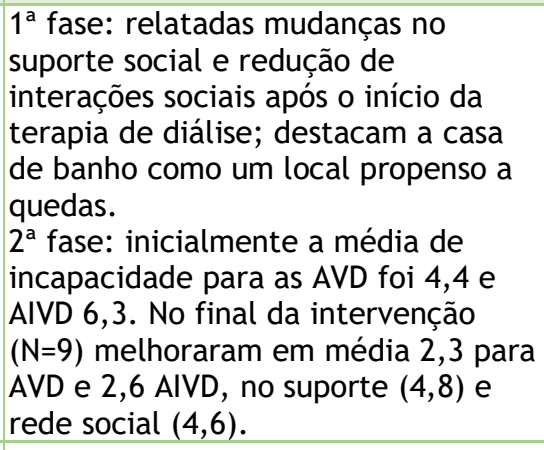 & $\begin{array}{l}\text { Aumento da funcionalidade e } \\
\text { da participação, através de } \\
\text { uma abordagem na pessoa e } \\
\text { no seu ambiente físico } \\
\text { domiciliar. }\end{array}$ \\
\hline França et al. & $\begin{array}{l}\text { Medir a qualidade de } \\
\text { vida de adultos com } \\
\text { lesão medular } \\
\text { (WHOQOL-bref); } \\
\text { Identificar os domínios } \\
\text { que prejudicam a } \\
\text { qualidade de vida. }\end{array}$ & $\begin{array}{l}47 \text { pessoas com } \\
\text { lesão medular. }\end{array}$ & $\begin{array}{l}\text { Os itens que apresentaram maior } \\
\text { comprometimento foram: domínio } \\
\text { físico locomoção e trabalho ( } 55,3 \% \\
\text { respetivamente); domínio psicológico } \\
\text { aproveitar a vida ( } 34,1 \%) \text {; no domínio } \\
\text { relações sociais demonstrou baixo } \\
\text { comprometimento nas relações } \\
\text { pessoais ( } 17 \%) \text { e apoio dos amigos } \\
(21,3 \%) \text { e no domínio ambiental todos } \\
\text { os itens apresentaram valores de } \\
\text { insatisfação. }\end{array}$ & $\begin{array}{l}\text { Necessidade de reformular } \\
\text { políticas públicas e } \\
\text { reorganizar os serviços de } \\
\text { saúde. } \\
\text { Os cuidados de enfermagem } \\
\text { na reabilitação e inclusão da } \\
\text { pessoa com lesão medular } \\
\text { devem atender às capacidades } \\
\text { individuais, desenvolver um } \\
\text { processo educativo } \\
\text { envolvendo o doente e seus } \\
\text { familiares. }\end{array}$ \\
\hline $\begin{array}{l}\text { Granbom et } \\
\text { al. }\end{array}$ & $\begin{array}{l}\text { Avaliar os efeitos da } \\
\text { intervenção gestão de }\end{array}$ & 153 idosos & $\begin{array}{l}\text { Na participação social os resultados } \\
\text { não mostraram diferenças }\end{array}$ & $\begin{array}{l}\text { Apesar de alguns resultados } \\
\text { estatisticamente significativos }\end{array}$ \\
\hline
\end{tabular}




\begin{tabular}{|c|c|c|c|c|}
\hline & $\begin{array}{l}\text { caso em idosos frágeis } \\
\text { a viver no domicílio, na } \\
\text { participação social e } \\
\text { atividades de lazer. }\end{array}$ & $\begin{array}{l}\text { Grupo de } \\
\text { intervenção }(n=80) \\
\text { Grupo de controlo } \\
(n=73)\end{array}$ & $\begin{array}{l}\text { significativas. Na realização de } \\
\text { atividades de lazer verificou-se uma } \\
\text { diferença estatisticamente } \\
\text { significativa no grupo de intervenção } \\
\text { aos } 3 \text { meses, e aos } 3 \text { e } 9 \text { meses } \\
\text { realizou mais atividades ao ar livre e } \\
\text { praticou exercício físico. }\end{array}$ & $\begin{array}{l}\text { a favor da intervenção gestão } \\
\text { de caso para a promoção da } \\
\text { participação social e em } \\
\text { atividades de lazer de idosos } \\
\text { frágeis que vivem na } \\
\text { comunidade são necessários } \\
\text { mais estudos nesta área. }\end{array}$ \\
\hline $\begin{array}{l}\text { Machado et } \\
\text { al. }\end{array}$ & $\begin{array}{l}\text { Refletir sobre as } \\
\text { implicações da Rede de } \\
\text { Cuidados da Pessoa } \\
\text { com Deficiência no } \\
\text { âmbito do Sistema } \\
\text { Único de Saúde. }\end{array}$ & $\begin{array}{l}\text { A Política Nacional } \\
\text { de Saúde da Pessoa } \\
\text { com Deficiência e } \\
\text { literatura relevante } \\
\text { sobre o tema }\end{array}$ & $\begin{array}{l}\text { A Rede de Cuidados da Pessoa com } \\
\text { Deficiência, não está totalmente } \\
\text { implementada existindo uma } \\
\text { desarticulação entre cada ponto de } \\
\text { atuação e a que os profissionais de } \\
\text { saúde prestem cuidados } \\
\text { isoladamente. Necessidade de } \\
\text { investimento na articulação da rede. }\end{array}$ & $\begin{array}{l}\text { Contribui para que os } \\
\text { profissionais que atuam na } \\
\text { área possuam competências, e } \\
\text { conhecimentos acerca das } \\
\text { pessoas com deficiência. }\end{array}$ \\
\hline $\begin{array}{l}\text { Nascimento } \\
\text { et al. }\end{array}$ & $\begin{array}{l}\text { Conhecer a } \\
\text { acessibilidade de } \\
\text { pessoas com } \\
\text { deficiência da } \\
\text { residência à unidade de } \\
\text { saúde da família. }\end{array}$ & $\begin{array}{l}\text { Roteiros de } \\
\text { observação das } \\
\text { condições de } \\
\text { acessibilidade } \\
\text { desde o domicílio à } \\
\text { unidade de saúde. }\end{array}$ & $\begin{array}{l}\text { Verificou-se que o percurso da } \\
\text { residência das pessoas com } \\
\text { deficiência à unidade de saúde, } \\
\text { possui muitos obstáculos que podem } \\
\text { inviabilizar a circulação. } \\
\text { Perda de autonomia e necessidade do } \\
\text { auxílio de terceiros. }\end{array}$ & $\begin{array}{l}\text { Os enfermeiros possuem papel } \\
\text { fundamental no } \\
\text { estabelecimento de vínculos, } \\
\text { podem traçar estratégias para } \\
\text { minimizar obstáculos, e ao } \\
\text { mesmo tempo aliviar } \\
\text { momentos de angústia e dor. }\end{array}$ \\
\hline Neiseh et al. & $\begin{array}{l}\text { Explorar quais são os } \\
\text { fatores facilitadores e } \\
\text { as barreiras do } \\
\text { processo de } \\
\text { emancipação de } \\
\text { pessoas com } \\
\text { deficiência física. }\end{array}$ & $\begin{array}{l}\text { Pessoas com } \\
\text { deficiência física } \\
(n=12), \text { Familiares } \\
(n=2), \text { Enfermeiro } \\
(n=1), \text { Terapeuta } \\
(n=1) \text { e Assistentes } \\
\text { sociais }(n=2)\end{array}$ & $\begin{array}{l}\text { No processo de emancipação } \\
\text { identificaram } 4 \text { barreiras (ambiente } \\
\text { envolvente e comunidade; } \\
\text { passividade das organizações; } \\
\text { interação entre pessoa-família; } \\
\text { autoconceito de deficiência } \\
\text { percecionado) e, } \\
4 \text { fatores facilitadores (redes sociais; } \\
\text { políticas; emancipação da família e } \\
\text { competências individuais). }\end{array}$ & $\begin{array}{l}\text { Através da compreensão e } \\
\text { identificação dos fatores que } \\
\text { afetam o processo de } \\
\text { emancipação das pessoas com } \\
\text { deficiência física contribui } \\
\text { para a prática de cuidados de } \\
\text { enfermagem, equipas de } \\
\text { reabilitação e na formulação } \\
\text { de políticas inclusivas. }\end{array}$ \\
\hline $\begin{array}{l}\text { Pereira et } \\
\text { al. }\end{array}$ & $\begin{array}{l}\text { Compreender se a } \\
\text { eliminação de barreiras } \\
\text { arquitetónicas é uma } \\
\text { preocupação dos } \\
\text { enfermeiros; Analisar } \\
\text { as diferenças entre os } \\
\text { enfermeiros de } \\
\text { reabilitação e os outros } \\
\text { enfermeiros. }\end{array}$ & 56 enfermeiros & $\begin{array}{l}\text { 60,7\% dos enfermeiros não sabe a } \\
\text { quem se dirigir para eliminar } \\
\text { barreiras arquitetónicas e } 58,9 \% \text { não } \\
\text { desenvolve nenhum tipo de } \\
\text { intervenção para tal. Os enfermeiros } \\
\text { de reabilitação possuem mais } \\
\text { conhecimento sobre: legislação } \\
\text { específica para a acessibilidade } \\
\text { ( } p=0,000) \text { e, sabem a quem se dirigir } \\
\text { para solicitar a eliminação de } \\
\text { barreiras arquitetónicas na } \\
\text { comunidade }(p=0,024) \text {. }\end{array}$ & $\begin{array}{l}\text { Na prestação de cuidados de } \\
\text { enfermagem a acessibilidade } \\
\text { e a eliminação de barreiras } \\
\text { arquitetónicas devem ser } \\
\text { prática diária dos } \\
\text { enfermeiros, no sentido de } \\
\text { promover a inclusão social das } \\
\text { pessoas com deficiência. }\end{array}$ \\
\hline
\end{tabular}

O conceito de pessoa com mobilidade condicionada também é aplicado e estas são definidas como aquelas que se deslocam em cadeira de rodas, que são incapazes de andar ou percorrer grandes distâncias, que apresentam dificuldades sensoriais ou que se encontram temporariamente condicionadas, como os idosos. $(24,25)$

Avaliar a capacidade funcional da pessoa com deficiência para a realização de atividades de vida e participação social

A Classificação Internacional de Funcionalidade, Incapacidade e Saúde pode ajudar neste processo contribuindo com padrões internacionais relativos às informações fornecidas e com conjuntos de perguntas que avaliam a situação de saúde e a experiência de deficiência, podendo ser utilizada para a construção das questões orientadoras. (23)

0 nível de independência da pessoa com deficiência, para a realização das atividades básicas de vida diária foi avaliado com recurso a escalas, como o índice de
Barthel e o índice de Katz. (13, 18, 20) Para avaliação da participação social foi utilizado o Modified Selfreport of Social Adjustment e o Reintegration to Normal Linving Index (18) e para avaliar as redes sociais a escala revista modificada Lubben Social Network e a escala de Krause and Borawski-Clark para avaliar o suporte recebido e satisfação/interações negativas com o suporte. (20)

Avaliar o impacto da alteração da capacidade funcional na qualidade de vida da pessoa

A lesão vertebro medular representa um problema de saúde pública no Brasil, porém os dados relativos à incidência de lesão medular são subnotificados, pelo que para a implementação de cuidados de enfermagem é premente conhecer as características da pessoa/população em que pretendemos intervir. (19)

Assim, no estudo de França, Coura (19) efetuaram um estudo epidemiológico com o objetivo de medir a qualidade de vida em pessoas com lesão vertebro medular e identificar os domínios que prejudicam a 
qualidade de vida. Os domínios mais afetados negativamente foram o físico e o ambiental, nomeadamente ao nível da locomoção, trabalho, dinheiro, informações lazer, vida sexual e aproveitamento da vida.

Implementar cuidados de enfermagem baseados em modelos teóricos e no processo de enfermagem

Os cuidados de enfermagem devem ser sustentados em referenciais teóricos, fundamentando a intervenção dos enfermeiros através da melhor evidência possível. (25)

Numa abordagem integrativa do cuidar e da reabilitação da pessoa com deficiência surge o modelo Caring - Disability Creation Process no Canadá. O Disability Creation Process orienta a prática dos cuidados em reabilitação, enfatizando a influência que os fatores pessoais e ambientais têm na participação social das pessoas com deficiência, ao adicionar o "cuidar" - a essência da enfermagem - permite uma abordagem mais ampla e holística do processo de reabilitação e inclusão social. Assim, os enfermeiros são eles mesmos fatores facilitadores ou barreiras no processo de reabilitação com o qual a pessoa e família está em constante interação.

Segundo este modelo, o cuidado em enfermagem, deve estar alicerçado em valores humanísticos e altruístas, segundo o sistema de crenças próprio da pessoa, na aceitação e reconhecimento de sentimentos positivos ou negativos, na resolução de problemas, no estabelecimento de uma relação terapêutica de apoio e confiança, na transmissão de conhecimento e aprendizagem pessoal, um cuidado de enfermagem mediante as necessidades da pessoa e através da criação de um ambiente que promova o bem-estar da pessoa. (26)

O ambiente é um conceito meta paradigmático da enfermagem devido à interação da pessoa com o ambiente onde se insere (27), sendo que outras teóricas de enfermagem sustentam a prática profissional através da integração do ambiente e de um olhar holístico sobre o processo de reabilitação da pessoa com deficiência. (25)

A reabilitação é um processo que engloba diversas dimensões e que vai além da recuperação das funções perdidas, o seu foco deve ser a pessoa, mas também a família e a comunidade uma vez que estão em constante interação, não sendo possível intervir sem o conhecimento de todo o meio envolvente como parte integrante do processo de reabilitação. (27)

Identificar barreiras arquitetónicas nos vários contextos de vida da pessoa e orientar para a sua eliminação

A experiência da deficiência, vai para além da capacidade funcional da pessoa, ela é exacerbada quando a pessoa com deficiência interage com o ambiente que a envolve, nomeadamente as condições da habitação e a existência de barreiras arquitetónicas no meio físico, a falta de acessibilidade ao meio edificado condicionando a inclusão e participação social. (20, 23-25, 27-29)

A identificação de barreiras arquitetónicas dentro da habitação da pessoa, a orientação para a sua reparação e modificação, o aconselhamento para a instalação de elementos arquitetónicos facilitadores, por exemplo as barras de apoio no sanitário, é um cuidado de enfermagem que permite a realização das atividades de vida de forma o mais independente possível e a participação social das pessoas com deficiência física adquirida. É igualmente importante ensinar, instruir e treinar para a realização dos vários autocuidados de acordo com as condições habitacionais de cada pessoa. $(20,23)$

A intervenção dos enfermeiros nos espaços de uso público é também ela uma oportunidade uma vez que é inegável a assimetria que existe relativamente às condições de acessibilidade, pois alguns destes espaços garantem todas as condições de acessibilidade enquanto que outros permanecem não acessíveis impossibilitando a realização das atividades e o desenvolvimento da vida diária, nomeadamente, bibliotecas, cafés, supermercados, bancos, igrejas/locais de culto, serviços de saúde, entre outros, prejudicando a igualdade de oportunidades, favorecendo a discriminação e acentuando a deficiência. $(24,25,27,28)$.

A prática desportiva para pessoas com deficiência fomenta a sua participação e inclusão social. Contudo, a existência de barreiras arquitetónicas nos edifícios desportivos são um entrave à sua realização, pelo que quando os enfermeiros aconselham à realização de atividade física devem fazê-lo de acordo com as características individuais da pessoa com deficiência e as condições de acessibilidade dos edifícios desportivos. (27)

Implementar programas de treino motor e de reabilitação social

A implementação de programas que se focam tanto na componente física como na ambiental, por exemplo através do fortalecimento muscular, e da identificação das redes sociais/isolamento ajuda a reduzir sintomatologia referida pelas pessoas com deficiência, por exemplo a fadiga e, consequentemente, a aumentar a sua participação social. (20)

O conceito de reabilitação social é introduzido por Portillo e Cowley (18) como sendo um processo dinâmico e subjetivo, podendo iniciar-se quer no hospital quer na comunidade, e no qual a pessoa se consciencializa para as suas limitações em termos de participação social e nesse sentido desenvolve atividades para promover a sua participação social nos vários contextos da vida em sociedade.

A reabilitação social deve ser encarada como um processo a longo prazo no qual alguns elementos desempenham um papel essencial: ambiente, atividades, interação social, autoconhecimento e consciência, enfrentamento e satisfação. (18) 
Conceber planos de cuidados personalizados - 0 Enfermeiro gestor de caso

O envolvimento das pessoas com deficiência física adquirida e das suas famílias no seu processo de reabilitação permite a prestação de cuidados personalizada e a conceção de um programa de cuidados de enfermagem desenhado à medida das necessidades e objetivos individuais, nomeadamente ao nível da participação e inclusão social.

Assim, Granbom, Kristensson (21) avaliaram os efeitos da intervenção modelo de gestão de caso em idosos frágeis a viver no domicílio, quanto à participação social e atividades de lazer. Os cuidados foram desenvolvidos através de visitas ao domicílio das pessoas e as intervenções aplicadas foram a gestão de caso tradicional, a transmissão de informação geral, por exemplo, sobre sistema de saúde, atividade física e social e transmissão de informação específica, sobre necessidades individuais de saúde, medicação e, segurança.

Promover e facilitar transições de cuidado seguras e eficazes através do empoderamento da pessoa com deficiência

Muitas pessoas com deficiência física adquirida necessitam de um cuidador para supervisionar ou ajudar na realização de atividades diárias. (23) Os familiares são considerados elementos fundamentais no processo de reabilitação no sentido em que incentivam as relações sociais fora do seio familiar e promovem o autocuidado, mas também devem ser eles mesmo foco de atenção dos enfermeiros para que os incentivem à realização de atividades sociais eles mesmos. (18)

É fundamental que a pessoa com deficiência tenha uma rede social de apoio composta por amigos, familiares, vizinhos, profissionais de saúde, governos locais, entre outros. $(19,24)$

Um outro aspeto importante de salientar é a necessidade de planeamento da alta hospitalar na transição de cuidados após a aquisição de uma deficiência física uma vez que é um aspeto crítico da prestação de cuidados quando pretendemos a inclusão social da pessoa com deficiência. $(13,22)$

Para o sucesso do processo de reabilitação e inclusão social da pessoa com deficiência as pessoas com deficiência e os seus familiares devem ser ensinados, instruídos e treinados para a continuidade dos cuidados inerentes sendo importante existirem roteiros e guias orientadores de planeamento da alta hospitalar de acordo com as necessidades individuais. $(13,22)$

Torna-se, também essencial que os enfermeiros conheçam os fatores que influenciam o processo de emancipação que permite aumentar a autonomia e a qualidade de vida destas pessoas. (29)

As pessoas com deficiência identificam como barreiras à sua emancipação a falta de suporte das comunidades, a passividade das organizações, as relações familiares vulneráveis e a autoperceção da deficiência. Por outro lado, os fatores que facilitam este processo são os ambientes flexíveis (através das redes sociais e internet), os programas de apoio (medidas legislativas e organizações não governamentais), ter uma família encorajadora do processo de emancipação e competências e características individuais. (29)

Os enfermeiros têm uma oportunidade para facilitar o processo de emancipação da pessoa com deficiência física, ao intervirem ao nível da eliminação de barreiras arquitetónicas no ambiente urbano, na sensibilização das comunidades para os direitos das pessoas com deficiência, ao nível da família na perceção da deficiência e nas decisões políticas sobre os direitos das pessoas com deficiência. (29)

Através do empoderamento da pessoa com deficiência, o enfermeiro, intervém ao nível da autonomia por forma a que as limitações funcionais que a pessoa possua em interação com o meio envolvente não a impeça do seu direito ao exercício de cidadania. (25)

Contribuir para o desenvolvimento de políticas, melhorando assistência à pessoa com deficiência e melhorando os serviços comunitários / colaboração com o poder autárquico

Os enfermeiros têm como função atuar como atores políticos, impulsionando políticas de saúde e decisões políticas, técnicas e sociais pela promoção da acessibilidade e inclusão social através da construção de territórios inclusivos valorizando a diversidade humana. $(24,25,28)$

A promoção da acessibilidade arquitetónica para a inclusão da pessoa com deficiência tem sido uma preocupação ao nível político. É fundamental que os enfermeiros articulem a sua prestação de cuidados com as autarquias locais para poderem em conjunto trabalhar na eliminação de barreiras arquitetónicas, na via pública e edifícios que recebem público, uma vez que condicionam a realização de atividades de vida diárias e a participação em atividades de lazer, lúdicas, desportivas, entre outras, tendo em vista a inclusão social da pessoa com deficiência. $(24,27)$

Os enfermeiros, nomeadamente, os enfermeiros de reabilitação uma vez que conhecem melhor as necessidades das pessoas com deficiência e, possuem conhecimento sobre legislação em vigor podem dar formação ao nível dos decisores políticos e das comunidades para sensibilizarem para práticas inclusivas e a eliminação de barreiras arquitetónicas promovendo a acessibilidade e inclusão social da pessoa com deficiência física $(24,25)$ dado que a falta de reconhecimento das necessidades destas pessoas quer pela população quer pelos governos é também um obstáculo para a mudança. (29)

A pessoa com deficiência, para além das necessidades de saúde associadas ao processo de reabilitação pode necessitar de outros cuidados diferenciados pelo que é necessária uma rede de cuidado integral que permita a continuidade assistencial à pessoa com deficiência, uma vez que aquilo que se assiste é a um cuidado fragmentado e desarticulado entre cada ponto de atuação, conduzindo à prestação de cuidados isolados em cada espaço de atuação comprometendo o processo de reabilitação. É indispensável que os profissionais 
sejam capacitados e possuam conhecimentos específicos sobre a prestação de cuidados às pessoas com deficiência para a inclusão e participação social (22) através da identificação de metas e objetivos satisfatórios para a pessoa, família e comunidade. (13, 22)

$\mathrm{Na}$ implementação destes princípios essenciais do cuidar em enfermagem, os enfermeiros contribuem para que a pessoa com deficiência física adquirida desenvolva o seu potencial máximo para alcançar a participação e inclusão social.

\section{CONCLUSÃO}

A presente revisão integrativa da literatura revelou que o tema da inclusão e participação social nos cuidados de enfermagem tem vindo a ser explorado nos últimos anos, principalmente no Brasil e em Portugal.

Acreditamos que esta revisão permite uma melhor compreensão sobre quais os cuidados de enfermagem são prestados e que têm como objetivo a inclusão social da pessoa com deficiência física adquirida.

A prestação dos cuidados de enfermagem à pessoa com deficiência deve estar alicerçada nos referenciais teóricos e na prática avançada em enfermagem no sentido de fornecer orientações para a prática. Para tal, é indispensável a avaliação e diagnóstico das necessidades e características das pessoas com deficiência, uma vez que se trata de uma população heterogénea e com características e necessidades individuais específicas.

Os cuidados de enfermagem para a inclusão social devem ter uma abordagem centrada na pessoa com deficiência física adquirida e sua família, prestando cuidados de enfermagem holísticos numa vertente biopsico-social, o que vai muito além da componente física, através da conceção de planos de cuidados personalizados e atendendo aos objetivos individuais da pessoa, às suas preferências e vontades, e ao envolvimento no seu próprio processo de reabilitação, alicerçando o cuidado no suporte familiar.

A prestação dos cuidados de enfermagem devem ser alargados para além da componente física e ter como foco os fatores ambientais, pela identificação de barreiras arquitetónicas, quer ao nível do ambiente domiciliar quer nos restantes ambientes da vida diária, que condicionam a vivência da deficiência e impedem a participação e inclusão social.

O conhecimento sobre a legislação em vigor e políticas de saúde é crucial para o cuidado de enfermagem podendo os enfermeiros trabalhar em colaboração com a pessoa com deficiência, família, outros profissionais e organizações, como autarquias locais influenciando as políticas de saúde, promovendo os melhores cuidados para a inclusão social da pessoa com deficiência.

\section{Limitações do estudo}

Destacamos que em grande parte os estudos identificados são de natureza descritiva sendo necessário mais estudos com níveis de evidência mais elevados, como os randomizados, para o fortalecimento da disseminação do conhecimento sobre a temática em análise.

Como constatámos em cada país a abordagem para avaliar a deficiência varia e isso influencia a capacidade para interpretar os resultados e efetuar comparações internacionais sendo necessário uma harmonização e padronização na forma de mensurar a deficiência. Ou seja, os dados devem ser relevantes e comparáveis ao nível global. Acresce que as realidades do contexto de cada país, de onde são provenientes os estudos, são bastante diferentes entre si.

\section{Implicações para a prática}

É importante realizar estudos que se foquem na promoção da capacidade funcional mas também que avaliem e desenvolvam intervenções de enfermagem e programas de reabilitação com o objetivo de promover a participação e inclusão social das pessoas com deficiência física adquirida, uma vez que muitos programas de reabilitação avaliam a participação social como um resultado, no entanto, não realizam nenhum tipo de intervenção específica nesse sentido.

\section{REFERÊNCIAS BIBLIOGRÁFICAS}

1. Resolução da Assembleia da República n. ${ }^{\circ}$ 56/2009, Aprova a Convenção sobre os Direitos das Pessoas com Deficiência, N. ${ }^{\circ} 146$. Sect. Diário da República: 1 . $^{\text {a }}$ série (2009).

2. Holanda CM, Andrade FLd, Bezerra MA, Nascimento JP, Neves Rda $F$, Alves SB, et al. Redes de apoio e pessoas com deficiência física: inserção social e acesso aos serviços de saúde. Cien Saude Colet [Internet]. 2015 Jan; 20(1):[175-184 pp.]. Disponível em: https://www.ncbi.nlm.nih.gov/pubmed/25650611.

3. Kim KM, Shin YR, Yu DC, Kim DK. The Meaning of Social Inclusion for People with Disabilities in South Korea. International Journal of Disability, Development and Education [Internet]. 2017; 64(1):[19-32 Dp.]. Disponível em: https://www.tandfonline.com/doi/citedby/10.1080/1034912X.2 016.1165802?scroll=top\&needAccess=true.

4. Cobigo V, Brown R, Lachapelle Y, Lysaght R, Martin L, OuelletteKuntz $\mathrm{H}$, et al. Social Inclusion: A Proposed Framework to Inform Policy and Service Outcomes Evaluation. Inclusion [Internet]. 2016; 4(4):[226-238 pp.]. Disponível em: https: / /meridian.allenpress.com/inclusion/article/4/4/226/261 /Social-Inclusion-A-Proposed-Framework-to-Inform.

5. Schoeller SD, Bento LM, Lorenzetti J, Pires D, Klein AC. Processo de trabalho em reabilitação: a perspectiva do trabalhador e do usuário. Aquichan [Internet]. 2015; 15(3):[403-412 pp.]. Disponível em: https: / / aquichan.unisabana.edu.co/index.php/aquichan/article /view/4233/3987.

6. Brignol P, Schoeller SD, Silva DMGVd, Boell JEW, Lopes SGR, Lim DKS. Viver com deficiência física e o papel da rede de apoio. Revista de Enfermagem do Centro- Oeste Mineiro [Internet]. 2018; $8 . \quad$ Disponível em: http://seer.ufsj.edu.br/index.php/recom/article/view/1957.

7. INE, PORDATA. População residente com deficiência segundo os Censos: total e por tipo de deficiência (2001) PORDATA: Base de Dados Portugal Contemporâneo; 2001 [updated 26-06-2015. Disponível

https://www.pordata.pt/Portugal/Popula\%C3\%A7\%C3\%A30+resid ente+com+defici\%C3\%AAncia+segundo+os+Censos+total+e+por+ti po+de+defici\%C3\%AAncia+(2001)-1239.

8. INE. Saúde e Incapacidades em Portugal 2011. Lisboa: Instituto Nacional de Estatística; 2012. Disponível em: 
https://www.ine.pt/ngt_server/attachfileu.jsp?look_parentBoui =149447974\&att_display=n\&att_download=y.

9. Valença TDC, Lima PV, Chaves RN, Santana EdS, Reis LAd. Repercussões sociais da aquisição de uma deficiência física na vida de idosos. Revista Kairós Gerontologia [Internet]. 2017; 20(1):[41-55 Pp.]. Disponível em: https://revistas. pucsp.br/kairos/article/view/2176-

901X.2017v20i1p41-55/22114.

10. Organização Mundial de Saúde. Classificação Internacional de Funcionalidade, Incapacidade e Saúde 2004. Disponível em: https://www.dgs.pt/estatisticas-de-saude/documentos-paradownload/classificacao-internacional-de-funcionalidadeincapacidade-e-saude-cif.aspx.

11. Organização Mundial de Saúde. Health Topics - Disabilities 2020 [Disponível http://origin.searo.who.int/topics/disabilities/en/.

12. Machado WCA, Silva HFd, Almeida WGd, De Figueiredo NMA, Martins MM, Henriques FMD, et al. Autocuidado para pessoas com deficiência adquirida: reflexão sobre intervenções de enfermagem frente aos enfrentamentos da reabilitação. Enfermagem em Foco. 2019;10(5).

13. Portillo MC, Cowley S. Working the way up in neurological rehabilitation: The holistic approach of nursing care. Journal of Clinical Nursing [Internet]. 2011; 20(11-12):[1731-1743 pp.]. Disponível

https: / /www.scopus.com/inward/ record.uri?eid=2-s2.0-

79955755669\&doi=10.1111\%2fj.1365-

2702.2010.03379.x\&partnerID=40\&md5=0d9db5e9c01845f211776

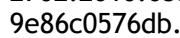

14. Alvarez AB, Machado WCA, Teixeira MLdO, Castelo Branco EMdS, Almeida de Figueiredo NM. Imagem corporal de paraplégicos: o enfrentamento das mudanças na perspectiva de pessoas com lesão medular [Body image in paraplegics: coping with changes from the perspective of people with spinal cord injury]. Revista Enfermagem UERJ. 2016;24(1). Disponível em: https://www.epublicacoes.uerj.br/index.php/enfermagemuerj/article/view/1 $6125 / 17872$.

15. Sousa LMd, Marques-Vieira CMA, Severino SSP, Antunes AV. A Metodologia de Revisão Integrativa da Literatura em Enfermagem. Revista de Investigação em Enfermagem [Internet]. 2017:[17-26 pp.]. Disponível em: https://repositoriocientifico.essatla.pt/bitstream/20.500.12253/1311/1/Metodolog ia\%20de\%20Revis\%c3\%a3o\%20Integrativa_RIE21_17-26.pdf.

16. Decreto-Lei n. ${ }^{\circ} 161 / 96$, Regulamento do Exercício Profissional dos Enfermeiros, $n^{\circ}$ 205. Sect. Diário da República: 1. ${ }^{a}$ série (1996).

17. Pereira RSDS, Martins MM, Gomes B, Laredo-Aguilera JA, Santos J. A intervenção do enfermeiro de reabilitação na promoção da acessibilidade. Revista Portuguesa de Enfermagem de Reabilitação. 2018;1(2):66-72. Disponível em: https://www.aper.pt/Ficheiros/Revista/RPERV1N2.pdf.

18. Portillo MC, Cowley S. Social rehabilitation in long-term conditions: Learning about the process. Journal of Advanced Nursing. 2011;67(6):1329-1340. Disponível em: https: //www. scopus.com/inward/record. uri?eid=2-s2.079955741305\&doi=10.1111\%2fj.1365-

2648.2010.05600. $x \&$ partnerlD=40\&md5=46b0967b5e333535ecd2f adf16769c25

https://onlinelibrary.wiley.com/doi/pdfdirect/10.1111/j.13652648.2010.05600.x?download=true.

19. França ISXd, Coura AS, Sousa FSd, Almeida PCd, Pagliuca LMF. Qualidade de vida em pacientes com lesão medular. Revista Gaúcha de Enfermagem. 2013;34(1):155-163. Disponível em: <Go to ISI>://MEDLINE:23781737.

20. Crews DC, Delaney AM, Walker Taylor JL, Cudjoe TKM, Nkimbeng $M$, Roberts $L$, et al. Pilot Intervention Addressing Social Support and Functioning of Low Socioeconomic Status Older Adults With ESRD: The Seniors Optimizing Community Integration to Advance Better Living with ESRD (SOCIABLE)Study. Kidney Medicine [Internet]. 2019; 1(1):[13-20 pp.]. Disponível em: https://www.sciencedirect.com/science/article/pii/S259005951 9300019.

21. Granbom M, Kristensson J, Sandberg M. Effects on leisure activities and social participation of a case management intervention for frail older people living at home: a randomised controlled trial. Health \& social care in the community [Internet]. 2017; 25(4):[1416-1429 pp.]. Disponível em: https: / / search.ebscohost.com/login. aspx?direct=true\&db=cmed m\&AN=28295847\&lang=pt-br\&site=ehost-live.

22. Machado WCA, Pereira JdS, Schoeller SD, Júlio LC, Martins MMFPdS, Figueiredo NMAd. Integralidade na rede de cuidados da pessoa com deficiência. Texto \& Contexto - Enfermagem. 2018;27(3). Disponível em: <Go to ISI>://SCIELO:S010407072018000300600.

23. Pires FRdO, Deitos BG, Schoeller SD, Santos SMAd, Vargas MAdO, Silva KM. Barreras y facilitadores arquitectónicos: un desafío para la independencia funcional. Index de Enfermería [Internet]. 2014; 23(3):[124-128 pp.]. Disponível em: http://scielo.isciii.es/scielo.php?script=sci_arttext\&pid=S113212962014000200002.

24. Pereira RSDS, Martins MM, Gomes BP, Schoeller SD, LaredoAguilera JA, Ribeiro I, et al. As autarquias e a promoção da acessibilidade arquitetónica. Revista de Enfermagem Referência. 2018; Série IV, n¹8:29-38. Disponível em: http: / /www.scielo.mec.pt/scielo.php?script=sci_arttext\&pid=S0 874-02832018000300004\&lang=pt.

25. Silva C, Oliveira F, Prazeres V, Ribeiro M, Ribeiro O. Novos desafios para velhos problemas- O Enfermeiro Especialista em Enfermagem de Reabilitação na Promoção da Acessibilidade. Revista Portuguesa de Enfermagem de Reabilitação. 2019;2:20 26.

26. St-Germain D, Boivin B, Fougeyrollas P. The Caring-Disability Creation Process model: a new way of combining 'Care' in nursing and 'Rehabilitation' for better quality of services and patient safety. Disability and Rehabilitation. 2011;33(21-22):2105-2113. Disponível em: <Go to ISI>://WOS:000295340600020 https://www.tandfonline.com/doi/abs/10.3109/09638288.2011. 560330.

27. Silva CS, Pacheco M, Martins MM, Carvalho AE, Pereira RSDS, Machado WCA. Acessibilidade dos Edifícios destinados à prática de desporto nas pessoas com deficiência. Revista Portuguesa de Enfermagem de Reabilitação. 2019;2:27-32.

28. Nascimento VFd, Gonçalves RA, Jesus WGd, Graça BCd, TerçasTrettel ACP, Hattori TY. Acessibilidade de Pessoas com Deficiência da Residência à Unidade de Saúde da Família. 2019;21(3):255-259. Disponível em: https: //search.ebscohost. com/login. aspx?direct=true\&db=a9h\& $\mathrm{AN}=139557407 \&$ lang=pt-br\&site=ehost-live https: //revista.pgsskroton.com/index.php/JHealthSci/article/d ownload/6123/4689.

29. Neiseh F, Dalvandi A, Tabrizi KN, Shahboulaghi FM, FallahiKhoshknab M, Shemshadi H. Barriers and Facilitators to Emancipation Process in Persons with Physical Disability-A Grounded Theory. Journal of Evolution of Medical and Dental Sciences-Jemds. 2020;9(16):1379-1385. Disponível em: < Go to ISI>://WOS:000535949900012. 